\title{
Perinephric Hematoma Following Ureteroscopy and Holmium Laser Lithotripsy
}

\author{
Branden G. Duffey ${ }^{*}$, Joseph Y. Lee and Manoj Monga \\ Department of Urology, University of Minnesota Medical Center, Minneapolis, Minnesota, USA
}

\begin{abstract}
We present a case of a ureteropyeloscopy with holmium laser lithotripsy for a $5 \mathrm{~mm}$ distal ureteral calculus that was complicated by perinephric hemorrhage despite utilization of a ureteral access sheath and the absence of any bleeding disorders. Conservative management was elected and the patient did not require transfusion of blood products. We believe this is the first case of perinephric hemorrhage under these circumstances and suggest that surgeons inform their patients of the low risk of bleeding complications with ureteroscopy.
\end{abstract}

Keywords: Urinary tract, calculi, lithotripsy, laser, hemorrhage.

\section{INTRODUCTION}

Improvements in ureteroscopic instruments and fiberoptics have enabled urologists to access the upper urinary tract in a relatively atraumatic fashion. In fact, ureteroscopy with small caliber instruments in conjunction with holmium laser lithotripsy has been shown to be safe even in patients susceptible to bleeding complications $[1,2]$. Herein we present a case of perinephric hematoma following ureteroscopy with holmium laser lithotripsy and stone basketing in a patient without any known bleeding diathesis. We believe this is the first report of perinephric hematoma under these conditions.

\section{CASE PRESENTATION AND MANAGEMENT}

A 19 year-old male without bleeding diathesis presented with right-sided flank pain. CT scan revealed a 4-5 mm distal right ureteral calculus with moderate hydroureteronephrosis. Repeat CT scan obtained after four weeks of observation showed failure of the calculus to pass and the patient elected to undergo ureteroscopy with stone extraction. There was no perinephric fluid collection or hematoma on any preoperative imaging studies. Preoperative serum hemoglobin was $17.5 \mathrm{~g} / \mathrm{dL}(13.3-17.7 \mathrm{~g} / \mathrm{dL})$, white blood cells $15.3 \times 10^{9} / \mathrm{L}$ $\left(4.0-11.0 \times 10^{9} / \mathrm{L}\right)$, platelets $262 \times 10^{9} / \mathrm{L}\left(150-450 \times 10^{9} / \mathrm{L}\right)$, and creatinine $1.2 \mathrm{mg} / \mathrm{dL}(0.6-1.2 \mathrm{mg} / \mathrm{dL})$. PT, PTT, and INR were in the normal range. Urinalysis and culture were negative.

The right ureteral orifice was cannulated with a $5 \mathrm{~F}$ openended ureteral catheter and a retrograde pyelogram was performed. A filling defect was seen in the distal ureter corresponding with known stone location. A 0.035 inch Bentson guide wire was passed through the $5 \mathrm{~F}$ open-ended ureteral catheter, easily circumnavigating the calculus and coiling in the renal pelvis. A Wolf $6.9 \mathrm{~F}$ semi-rigid ureteroscope was inserted into the bladder, but could not cannulate the ureteral orifice. Ureteral orifice dilatation was performed over the

*Address correspondence to this author at the Department of Urology, University of Minnesota Medical Center, MMC 394, 420 Delaware St. SE, Minneapolis, MN 55455, USA; Tel: 612-625-3209; Fax: 612-624-4430;

E-mail: duff0144@umn.edu safety wire with the obturator of a Cook Flexor 12/14F ureteral access sheath. After this maneuver the ureter was easily entered and ureteroscopy performed. Ureteroscopic inspection of the distal and mid ureter demonstrated that the calculus had migrated more proximally. A Superstiff guide wire was passed through the ureteroscope and coiled in the renal pelvis. A Cook Flexor 12/14F, $35 \mathrm{~cm}$ ureteral access sheath was placed over the super-stiff guidewire with the tip positioned about $2 \mathrm{~cm}$ above the sacrum. All maneuvers with guide wires and the access sheath were monitored with fluoroscopy; there was no evidence of wire or sheath trauma. Flexible ureteropyeloscopy was performed and the calculus was identified in the upper-pole posterior calyx. Hand irrigation with saline was carried out with a Boston-Scientific single-action pump. Holmium laser lithiotripsy with a $200 \mathrm{mi}-$ cron fiber set at 0.6 Joules/6 Hertz was accomplished, breaking the stone into three small fragments. One $4 \mathrm{~mm}$ and one 2 $\mathrm{mm}$ fragment were basketed and extracted with a $2.2 \mathrm{~F}$ nitinol tipless basket. One sub-millimeter fragment was not retrieved. Thorough pyeloscopy showed no identifiable injuries, no significant residual stones and a moderate blood clot in the renal pelvis. The ureter was inspected in its entirety as the ureteral access sheath and flexible ureteroscope were withdrawn en bloc; no ureteral injuries were identified. A 6 F x $26 \mathrm{~cm} \mathrm{JJ}$ ureteral stent was placed with excellent coil in the renal pelvis and urinary bladder. The bladder was decompressed with a foley catheter until his discharge home four hours later.

The patient returned to his local Emergency Department that evening complaining of severe right flank pain, nausea and vomiting. CT imaging showed a tract consistent with acute bleeding extending posteriorly from the upper-pole posterior calyx to a moderate sized perinephric hematoma. (Fig. 1) Serum hemoglobin was 16.2. His platelet count and coagulation parameters were normal. He was admitted for pain control and managed conservatively with bed rest, analgesics, and serial hemoglobins. Red blood cell transfusion was not necessary. He was discharged hospital day two with minimal pain and a serum hemoglobin of 15.3. 


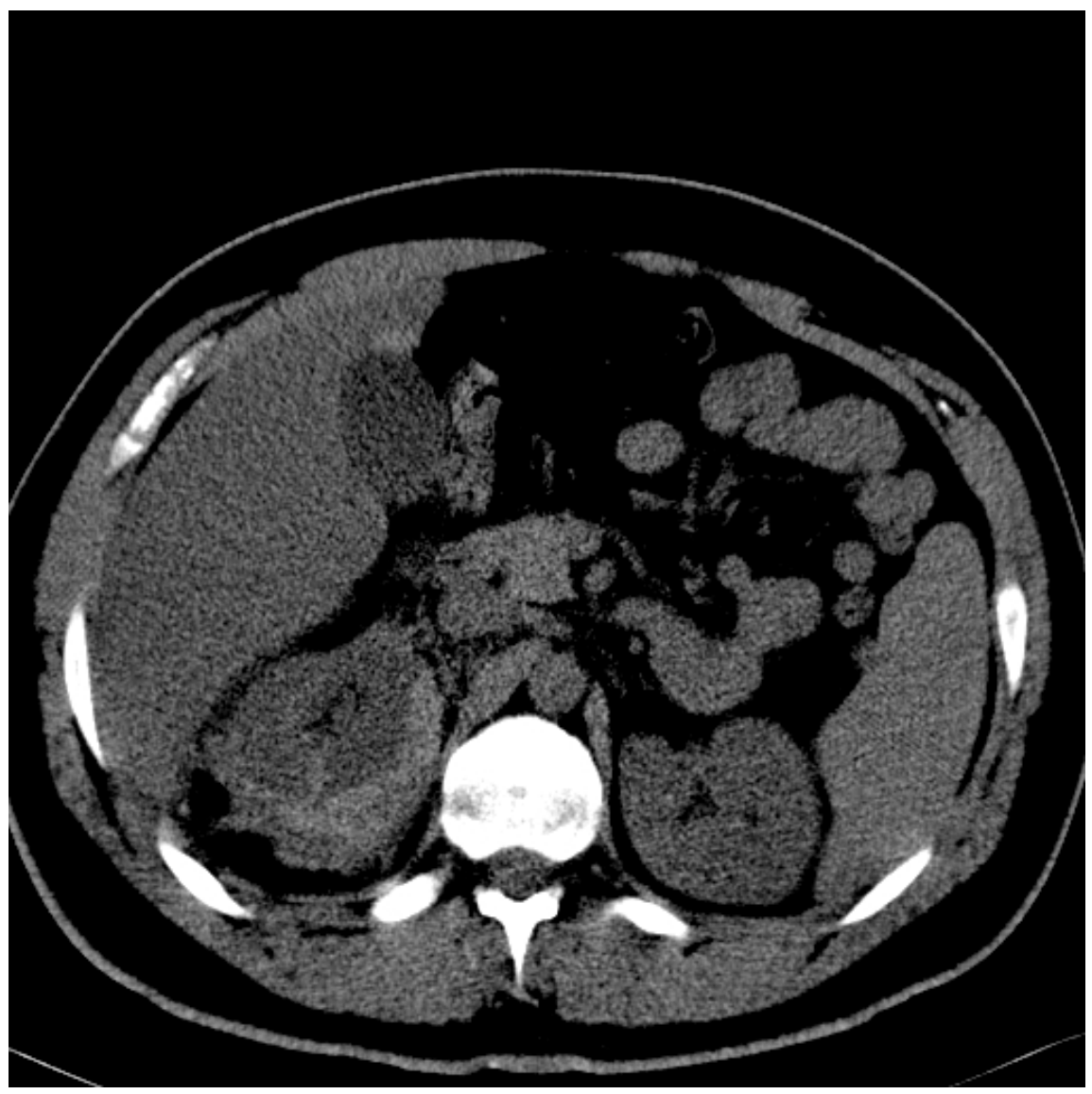

Fig. (1). CT abdomen/pelvis without contrast showing blood extending from a right posterior interpolar calyx to a perinephric hematoma.

\section{DISCUSSION}

Ureteroscopy has been reported to be a safe and efficacious alternative in patients who are anticoagulated or have bleeding diathesis [1,2]. In these series of 9 and 30 patients respectively, 2 procedure-related bleeding complications were reported; oliguria secondary to a small ureteral clot and significant retroperitoneal hemorrhage requiring transfusion after electrohydraulic lithotripsy. In the present case, a holmium laser was used for lithotripsy reducing the risk of injury to the urothelium and there were no visible injuries on final inspection of the collecting system. Given these factors, a possible etiology of the hemorrhage is forniceal rupture secondary to elevated irrigating pressures. Rehman et al. studied intrapelvic pressures in cadaveric collecting systems during ureteropyeloscopy with ureteral access sheaths and found that the 12/14 access sheath provided maximal irrigant flow while maintaining intrarenal pressures below $20 \mathrm{~cm} \mathrm{H}_{2} \mathrm{O}$ [3]. This study suggested that positioning of the ureteral access sheath at the renal pelvis may optimize intrarenal pressures during ureteropyeloscopy. In our procedure, the sheath was positioned in the mid-ureter. Though an access sheath was utilized, it is possible that vigorous irrigation in a single calyx could cause a transient increase in regional pressure resulting in forniceal rupture and subsequent perinephric hemorrhage.

\section{CONCLUSIONS}

This case suggests that bleeding should be included in the risk-benefit counseling during the informed consent process for ureteroscopy. We continue to view ureteroscopy with laser lithotripsy as having a low risk of bleeding, even in patients with bleeding diathesis, however recognize the potential for hemorrhagic complications and advocate irrigating at the lowest pressures feasible.

\section{REFERENCES}

[1] Watterson JD, Girvan AR, Cook AJ, et al. Safety and efficacy of holmium: YAG laser lithotripsy in patients with bleeding diatheses. J Urol 2002; 168(2): 442-5.

[2] Kuo RL, Aslan P, Fitzgerald KB, Preminger GM. Use of ureteroscopy and holmium: YAG laser in patients with bleeding diatheses. Urology 1998; 52(4): 609-13.

[3] Rehman J, Monga M, Landman J, et al. Characterization of intrapelvic pressure during ureteropyeloscopy with ureteral access sheaths. Urology 2003; 61(4): 713-8. 